\title{
Convergence of an extragradient-like iterative algorithm for monotone mappings and nonexpansive mappings
}

Yuan Qing ${ }^{1}$ and Meijuan Shang ${ }^{2,3^{*}}$

"Correspondence:

meijuanshang@yahoo.com.cn ${ }^{2}$ Department of Mathematics,

School of Science, Beijing Jiaotong University, Beijing, 100044, China

${ }^{3}$ Department of Mathematics,

Shijiazhuang University,

Shijiazhuang, 050035, China

Full list of author information is

available at the end of the article

\begin{abstract}
In this paper, we investigate the problem of finding some common element in the set of common fixed points of an infinite family of nonexpansive mappings and in the set of solutions of variational inequalities based on an extragradient-like iterative algorithm. Strong convergence of the purposed iterative algorithm is obtained. MSC: 47H05; 47H09; 47J25; 90C33
\end{abstract}

Keywords: inverse-strongly monotone mapping; fixed point; nonexpansive mapping; projection; strong convergence

\section{Introduction}

Iterative algorithms have been playing an important role in the approximation solvability, especially of nonlinear variational inequalities as well as of nonlinear equations in several fields such as mechanics, traffic, economics, information, medicine, and many others. The well-known convex feasibility problem which captures applications in various disciplines such as image restoration and radiation therapy treatment planning is to find a point in the intersection of common fixed point sets of a family of nonlinear mappings; see, for example, [1-11]. The Mann iterative algorithm is an efficient method to study the class of nonexpansive mappings. Indeed, Picard cannot converge even that the fixed point set of nonexpansive mappings is nonempty.

It is known that Mann iterative algorithm only has weak convergence for nonexpansive mappings in infinite-dimensional Hilbert spaces; see [12] for more details and the references therein. In many disciplines, including economics [13], image recovery [14], quantum physics [15-20], and control theory [21], problems arise in infinite dimension spaces. To improve the weak convergence of the Mann iterative algorithm, many authors considered using contractions to approximate nonexpansive mappings; for more details, see [22] and [23] and the references therein.

In this paper, we focus on the problem of finding some common element in the set of common fixed points of an infinite family of nonexpansive mappings and in the set of solutions of variational inequalities based on an extragradient-like iterative algorithm. Some deduced sub-results and applications are obtained.

(c) 2013 Qing and Shang; licensee Springer. This is an Open Access article distributed under the terms of the Creative Commons Attribution License (http://creativecommons.org/licenses/by/2.0), which permits unrestricted use, distribution, and reproduction in any medium, provided the original work is properly cited. 


\section{Preliminaries}

Throughout this paper, we assume that $H$ is a real Hilbert space whose inner product and norm are denoted by $\langle\cdot, \cdot\rangle$ and $\|\cdot\|$, respectively. Let $K$ be a nonempty, closed, and convex subset of $H$. Let $P_{K}$ be the metric projection from $H$ onto $K$.

Recall that a mapping $B: K \rightarrow H$ is said to be inverse-strongly monotone iff there exists a positive real number $\mu$ such that

$$
\langle B x-B y, x-y\rangle \geq \mu\|B x-B y\|^{2}, \quad \forall x, y \in K .
$$

For such a case, $B$ is also said to be $\mu$-inverse-strongly monotone.

Recall that a mapping $T: K \rightarrow K$ is said to be nonexpansive iff

$$
\|T x-T y\| \leq\|x-y\|, \quad \forall x, y \in K .
$$

In this paper, we use $F(T)$ to denote the fixed point set of the mapping $T$.

Recall that a mapping $f: K \rightarrow K$ is said to be a contraction iff there exists a coefficient $\alpha \in(0,1)$ such that

$$
\|f(x)-f(y)\| \leq \alpha\|x-y\|, \quad \forall x, y \in K .
$$

For such a case, $f$ is also said to be an $\alpha$-contraction.

Recall that a linear bounded operator $A: K \rightarrow K$ is strongly positive iff there exists a constant $\bar{\gamma}>0$ such that

$$
\langle A x, x\rangle \geq \bar{\gamma}\|x\|^{2}, \quad \forall x \in K .
$$

Recall that a set-valued mapping $S: H \rightarrow 2^{H}$ is said to be monotone iff $f \in S x$ and $g \in S y$ imply

$$
\langle x-y, f-g\rangle \geq 0, \quad \forall x, y \in H .
$$

A monotone mapping $S: H \rightarrow 2^{H}$ is maximal iff the graph of $G(S)$ of $S$ is not properly contained in the graph of any other monotone mapping. It is known that a monotone mapping $S$ is maximal iff for $(x, f) \in H \times H,\langle x-y, f-g\rangle \geq 0$ for every $(y, g) \in G(S)$ implies $f \in S x$. Let $Q: C \rightarrow H$ be a monotone mapping and $N_{K} v$ be the normal cone to $K$ at $v \in K$, i.e., $N_{K} v=\{w \in H:\langle v-u, w\rangle \geq 0, \forall u \in K\}$, and define

$$
S v= \begin{cases}Q v+N_{K} v, & v \in K, \\ \emptyset, & v \notin K .\end{cases}
$$

Then $S$ is maximal monotone and $0 \in S v$ iff $v \in V I(K, A)$; see [24] for more details.

Recall that the classical variational inequality is to find a $u \in K$ such that

$$
\langle B u, v-u\rangle \geq 0, \quad \forall v \in K
$$

where $B: K \rightarrow H$ is a monotone mapping. It is known that $u \in K$ is a solution to (2.1) iff $u$ is a fixed point of the mapping $P_{K}(I-\lambda B)$, where $\lambda>0$ is a constant and $I$ stands 
for the identity mapping. In this paper, we use $V I(K, B)$ to denote the solution set of the variational inequality (2.1).

Iterative algorithms for nonexpansive mappings have recently been applied to solve convex minimization problems. A typical problem is to minimize a quadratic function over the set of fixed points of a nonexpansive mapping $T$ on a real Hilbert space $H$,

$$
\min _{x \in F(T)} \frac{1}{2}\langle A x, x\rangle-\langle x, u\rangle
$$

where $A$ is a linear bounded self-adjoint operator on $H$ and $u$ is a given point in $H$. In [25], it is proved that the sequence $\left\{x_{n}\right\}$ defined by the iterative algorithm

$$
x_{0} \in H, \quad x_{n+1}=\left(I-\alpha_{n} A\right) T x_{n}+\alpha_{n} u, \quad n \geq 0,
$$

strongly converges to the unique solution of the minimization problem (2.2) provided that the sequence $\left\{\alpha_{n}\right\}$ satisfies certain restriction.

Recently, Marino and Xu [26] reconsidered the problem by viscosity approximation method. They investigated the following iterative algorithm:

$$
x_{0} \in H, \quad x_{n+1}=\left(I-\alpha_{n} A\right) T x_{n}+\alpha_{n} \gamma f\left(x_{n}\right), \quad n \geq 0,
$$

where $A$ is a linear bounded self-adjoint operator on $H, T: H \rightarrow H$ is a nonexpansive mapping, and $f: H \rightarrow H$ is a contraction. They proved that the sequence $\left\{x_{n}\right\}$ generated in the above iterative process converges strongly to the unique solution of the following variational inequality:

$$
\left\langle(A-\gamma f) x^{*}, x-x^{*}\right\rangle \geq 0, \quad x \in K,
$$

which is the optimality condition for the minimization problem

$$
\min _{x \in F(T)} \frac{1}{2}\langle A x, x\rangle-h(x)
$$

where $h$ is a potential function for $\gamma f$, that is, $h^{\prime}(x)=\gamma f(x)$ for $x \in H$.

Recently, the problem of finding a common element in the fixed point set of a nonexpansive mapping and in the solution set of a variational inequality has been considered by many authors; see, for example, [27-40] and the references therein. In 2003, Takahashi and Toyoda [35] considered the following iterative algorithm:

$$
x_{1} \in K, \quad x_{n+1}=\alpha_{n} x_{n}+\left(1-\alpha_{n}\right) T P_{K}\left(x_{n}-\lambda_{n} B x_{n}\right), \quad n \geq 1,
$$

where $T: K \rightarrow K$ is a nonexpansive mapping, $B: K \rightarrow H$ is a $\mu$-inverse-strongly monotone mapping, $\left\{\alpha_{n}\right\}$ is a sequence in $(0,1)$, and $\left\{\lambda_{n}\right\}$ is a sequence in $(0,2 \mu)$. They showed that the sequence $\left\{x_{n}\right\}$ generated in (2.3) weakly converges to some point $z \in F(T) \cap$ $V I(K, B)$.

Iiduka and Takahashi [36] reconsidered the common element problem via the following iterative algorithm:

$$
x_{1}=x \in K, \quad x_{n+1}=\alpha_{n} x+\left(1-\alpha_{n}\right) T P_{K}\left(x_{n}-\lambda_{n} B x_{n}\right), \quad n \geq 1,
$$


where $T: K \rightarrow K$ is a nonexpansive mapping, $B: K \rightarrow H$ is a $\mu$-inverse-strongly monotone mapping, $\left\{\alpha_{n}\right\}$ is a sequence in $(0,1)$, and $\left\{\lambda_{n}\right\}$ is a sequence in $(0,2 \mu)$. They proved that the sequence $\left\{x_{n}\right\}$ strongly converges to some point $z \in F(T) \cap V I(K, B)$.

In this paper, we will consider an infinite family of nonexpansive mappings. More precisely, we consider the mapping $W_{n}$ defined by

$$
\begin{aligned}
& U_{n, n+1}=I, \\
& U_{n, n}=\gamma_{n} T_{n} U_{n, n+1}+\left(1-\gamma_{n}\right) I, \\
& U_{n, n-1}=\gamma_{n-1} T_{n-1} U_{n, n}+\left(1-\gamma_{n-1}\right) I, \\
& \vdots \\
& U_{n, k}=\gamma_{k} T_{k} U_{n, k+1}+\left(1-\gamma_{k}\right) I, \\
& U_{n, k-1}=\gamma_{k-1} T_{k-1} U_{n, k}+\left(1-\gamma_{k-1}\right) I, \\
& \vdots \\
& U_{n, 2}=\gamma_{2} T_{2} U_{n, 3}+\left(1-\gamma_{2}\right) I, \\
& W_{n}=U_{n, 1}=\gamma_{1} T_{1} U_{n, 2}+\left(1-\gamma_{1}\right) I,
\end{aligned}
$$

where $\gamma_{1}, \gamma_{2}, \ldots$ are real numbers such that $0 \leq \gamma_{n} \leq 1, T_{1}, T_{2}, \ldots$ is an infinite family of mappings of $K$ into itself. Nonexpansivity of each $T_{i}$ ensures the nonexpansivity of $W_{n}$.

Regarding $W_{n}$, we have the following lemmas which are important to prove our main results.

Lemma 2.1 [41] Let $K$ be a nonempty, closed, and convex subset of a strictly convex Banach space $E$. Let $T_{1}, T_{2}, \ldots$ be nonexpansive mappings of $K$ into itself such that $\bigcap_{n=1}^{\infty} F\left(T_{n}\right)$ is nonempty, and let $\gamma_{1}, \gamma_{2}, \ldots$ be real numbers such that $0<\gamma_{n} \leq b<1$ for any $n \geq 1$. Then, for every $x \in K$ and $k \in N$, the limit $\lim _{n \rightarrow \infty} U_{n, k} x$ exists.

Using Lemma 2.1, one can define the mapping $W$ as follows:

$$
W x=\lim _{n \rightarrow \infty} W_{n} x=\lim _{n \rightarrow \infty} U_{n, 1} x, \quad \forall x \in K .
$$

Such a mapping $W$ is called $W$-mapping generated by $T_{1}, T_{2}, \ldots$ and $\gamma_{1}, \gamma_{2}, \ldots$

Throughout this paper, we will assume that $0<\gamma_{n} \leq b<1$ for each $n \geq 1$.

Lemma 2.2 [41] Let $K$ be a nonempty, closed, and convex subset of a strictly convex Banach space E. Let $T_{1}, T_{2}, \ldots$ be nonexpansive mappings of $K$ into itself such that $\bigcap_{n=1}^{\infty} F\left(T_{n}\right)$ is nonempty, and let $\gamma_{1}, \gamma_{2}, \ldots$ be real numbers such that $0<\gamma_{n} \leq b<1$ for each $n \geq 1$. Then $F(W)=\bigcap_{n=1}^{\infty} F\left(T_{n}\right)$.

In this paper, motivated by the above results, we investigate the problem of approximating a common element in the solution set of variational inequalities and in the common fixed point set of a family of nonexpansive mappings based on an extragradient-like iterative algorithm. Strong convergence theorems of common elements are established in the framework of Hilbert spaces. 
In order to prove our main results, we also need the following lemmas.

Lemma 2.3 In a real Hilbert space $H$, the following inequality holds:

$$
\|x+y\|^{2} \leq\|x\|^{2}+2\langle y, x+y\rangle, \quad \forall x, y \in H
$$

Lemma 2.4 [26] Assume $A$ is a strongly positive linear bounded self-adjoint operator on a Hilbert space $H$ with the coefficient $\bar{\gamma}>0$ and $0<\rho \leq\|A\|^{-1}$. Then $\|I-\rho A\| \leq 1-\rho \bar{\gamma}$.

Lemma 2.5 [26] Let $H$ be a Hilbert space. Let $A$ be a strongly positive linear bounded self-adjoint operator with the coefficient $\bar{\gamma}>0$. Assume that $0<\gamma<\bar{\gamma} / \alpha$. Let $T$ be a nonexpansive mapping with a fixed point $x_{t} \in H$ of the contraction $x \mapsto t \gamma f(x)+(I-t A) T x$. Then $\left\{x_{t}\right\}$ converges strongly as $t \rightarrow 0$ to a fixed point $\bar{x}$ of $T$, which solves the variational inequality

$$
\langle(A-\gamma f) \bar{x}, z-\bar{x}\rangle \leq 0, \quad \forall z \in F(T) .
$$

Equivalently, we have $P_{F(T)}(I-A+\gamma f) \bar{x}=\bar{x}$.

Lemma 2.6 [42] Assume that $\left\{\alpha_{n}\right\}$ is a sequence of nonnegative real numbers such that

$$
\alpha_{n+1} \leq\left(1-\gamma_{n}\right) \alpha_{n}+\delta_{n}
$$

where $\left\{\gamma_{n}\right\}$ is a sequence in $(0,1)$ and $\left\{\delta_{n}\right\}$ is a sequence such that

(a) $\sum_{n=1}^{\infty} \gamma_{n}=\infty$;

(b) $\limsup _{n \rightarrow \infty} \delta_{n} / \gamma_{n} \leq 0$ or $\sum_{n=1}^{\infty}\left|\delta_{n}\right|<\infty$.

Then $\lim _{n \rightarrow \infty} \alpha_{n}=0$.

Lemma 2.7 [39] Let $K$ be a nonempty closed convex subset of a Hilbert space $H,\left\{T_{i}\right.$ : $C \rightarrow C\}$ be a family of infinitely nonexpansive mappings with $\bigcap_{i=1}^{\infty} F\left(T_{i}\right) \neq \emptyset,\left\{\gamma_{n}\right\}$ be a real sequence such that $0<\gamma_{n} \leq b<1$ for each $n \geq 1$. If $C$ is any bounded subset of $K$, then $\lim _{n \rightarrow \infty} \sup _{x \in C}\left\|W x-W_{n} x\right\|=0$.

\section{Main results}

Theorem 3.1 Let $K$ be a nonempty, closed, and convex subset of a real Hilbert space H. Let $B_{i}: K \rightarrow H$ be $\mu_{i}$-inverse-strongly monotone mappings for each $i=1,2$, and $f: K \rightarrow K$ be an $\alpha$-contraction. Let $A: K \rightarrow K$ be a strongly positive linear bounded self-adjoint operator with the coefficient $\bar{\gamma}>0$. Let $\left\{x_{n}\right\}$ be a sequence generated in the following extragradientlike iterative algorithm:

$$
\left\{\begin{array}{l}
x_{1} \in K, \\
y_{n}=P_{K}\left(x_{n}-\eta_{n} B_{2} x_{n}\right), \\
x_{n+1}=P_{K}\left(\alpha_{n} \gamma f\left(x_{n}\right)+\left(I-\alpha_{n} A\right) W_{n} P_{K}\left(I-\lambda_{n} B_{1}\right) y_{n}\right), \quad n \geq 1,
\end{array}\right.
$$

where $P_{K}$ is the metric projection from $H$ onto $K, W_{n}$ is a mapping defined by (2.5), $\left\{\alpha_{n}\right\}$ is a real number sequence in $(0,1)$, and $\left\{\lambda_{n}\right\},\left\{\eta_{n}\right\}$ are two positive real number sequences. Assume that $F=\bigcap_{i=1}^{\infty} F\left(T_{i}\right) \cap V I\left(K, B_{1}\right) \cap V I\left(K, B_{2}\right) \neq \emptyset, 0<\gamma<\bar{\gamma} / \alpha$ and the following restrictions are satisfied: 
(a) $\lim _{n \rightarrow \infty} \alpha_{n}=0, \sum_{n=1}^{\infty} \alpha_{n}=\infty$, and $\sum_{n=1}^{\infty}\left|\alpha_{n+1}-\alpha_{n}\right| \leq \infty$;

(b) $\sum_{n=1}^{\infty}\left|\eta_{n+1}-\eta_{n}\right|<\infty, \sum_{n=1}^{\infty}\left|\lambda_{n+1}-\lambda_{n}\right|<\infty$;

(c) $\left\{\eta_{n}\right\},\left\{\lambda_{n}\right\} \subset[u, v]$, where $0<u<v<2 \min \left\{\mu_{1}, \mu_{2}\right\}$.

Then the sequence $\left\{x_{n}\right\}$ strongly converges to $x^{*} \in F$, where $x^{*}=P_{F}(\gamma f+(I-A)) x^{*}$.

Proof First, we show that $I-\lambda_{n} B_{1}$ and $I-\eta_{n} B_{2}$ are nonexpansive. Indeed, we see from the restriction (c) that

$$
\begin{aligned}
\left\|\left(I-\lambda_{n} B_{1}\right) x-\left(I-\lambda_{n} B_{1}\right) y\right\|^{2} & =\left\|x-y-\lambda_{n}\left(B_{1} x-B_{1} y\right)\right\|^{2} \\
& =\|x-y\|^{2}-2 \lambda_{n}\left\langle x-y, B_{1} x-B_{1} y\right\rangle+\lambda_{n}^{2}\left\|B_{1} x-B_{1} y\right\|^{2} \\
& \leq\|x-y\|^{2}+\lambda_{n}\left(\lambda_{n}-2 \mu_{1}\right)\left\|B_{1} x-B_{1} y\right\|^{2} \\
& \leq\|x-y\|^{2}, \quad \forall x, y \in C .
\end{aligned}
$$

This shows that $I-\lambda_{n} B_{1}$ is nonexpansive, so is $I-\eta_{n} B_{2}$. Noticing the condition (a), we may assume, with no loss of generality, that $\alpha_{n} \leq\|A\|^{-1}$ for each $n \geq 1$. It follows from Lemma 2.4 that $\left\|I-\alpha_{n} A\right\| \leq 1-\alpha_{n} \bar{\gamma}$.

Next, we show that the sequence $\left\{x_{n}\right\}$ is bounded. Letting $p \in F$, we see that

$$
\left\|y_{n}-p\right\|=\left\|P_{K}\left(I-\eta_{n} B_{2}\right) x_{n}-P_{K}\left(I-\eta_{n} B_{2}\right) p\right\| \leq\left\|x_{n}-p\right\| .
$$

It follows that

$$
\begin{aligned}
\left\|x_{n+1}-p\right\| & \leq\left\|\alpha_{n}\left(\gamma f\left(x_{n}\right)-A p\right)+\left(I-\alpha_{n} A\right)\left(W_{n} P_{C}\left(I-\lambda_{n} B\right) y_{n}-p\right)\right\| \\
& \leq \alpha_{n}\left\|\gamma f\left(x_{n}\right)-A p\right\|+\left(1-\alpha_{n} \bar{\gamma}\right)\left\|W_{n} P_{C}\left(I-\lambda_{n} B\right) y_{n}-p\right\| \\
& \leq \alpha_{n} \gamma\left\|f\left(x_{n}\right)-f(p)\right\|+\alpha_{n}\|\gamma f(p)-A p\|+\left(1-\alpha_{n} \bar{\gamma}\right)\left\|y_{n}-p\right\| \\
& =\left(1-\alpha_{n}(\bar{\gamma}-\gamma \alpha)\right)\left\|x_{n}-p\right\|+\alpha_{n}\|\gamma f(p)-A p\| .
\end{aligned}
$$

By simple induction, we have

$$
\left\|x_{n}-p\right\| \leq \max \left\{\left\|x_{1}-p\right\|, \frac{\|A p-\gamma f(p)\|}{\bar{\gamma}-\gamma \alpha}\right\},
$$

which yields that the sequence $\left\{x_{n}\right\}$ is bounded, so is $\left\{y_{n}\right\}$. Notice that

$$
\begin{aligned}
& \left\|y_{n+1}-y_{n}\right\| \\
& \quad=\left\|P_{K}\left(I-\eta_{n+1} B_{2}\right) x_{n+1}-P_{K}\left(I-\eta_{n} B_{2}\right) x_{n}\right\| \\
& \quad \leq\left\|\left(I-\eta_{n+1} B_{2}\right) x_{n+1}-\left(I-\eta_{n+1} B_{2}\right) x_{n}+\left(I-\eta_{n+1} B_{2}\right) x_{n}-\left(I-\eta_{n} B_{2}\right) x_{n}\right\| \\
& \quad \leq\left\|x_{n+1}-x_{n}\right\|+\left|\eta_{n+1}-\eta_{n}\right|\left\|B_{2} x_{n}\right\| .
\end{aligned}
$$

Putting $\rho_{n}=P_{K}\left(I-\lambda_{n} B_{1}\right) y_{n}$, we have

$$
\begin{aligned}
& \left\|\rho_{n+1}-\rho_{n}\right\| \\
& \quad=\left\|P_{K}\left(I-\lambda_{n+1} B_{1}\right) y_{n+1}-P_{K}\left(I-\lambda_{n} B_{1}\right) y_{n}\right\|
\end{aligned}
$$




$$
\begin{aligned}
& \leq\left\|\left(I-\lambda_{n+1} B_{1}\right) y_{n+1}-\left(I-\lambda_{n+1} B_{1}\right) y_{n}+\left(I-\lambda_{n+1} B_{1}\right) y_{n}-\left(I-\lambda_{n} B_{1}\right) y_{n}\right\| \\
& \leq\left\|y_{n+1}-y_{n}\right\|+\left|\lambda_{n+1}-\lambda_{n}\right|\left\|B_{1} y_{n}\right\| .
\end{aligned}
$$

Substituting (3.2) into (3.3), we arrive at

$$
\left\|\rho_{n+1}-\rho_{n}\right\| \leq\left\|x_{n+1}-x_{n}\right\|+M_{1}\left(\left|\eta_{n+1}-\eta_{n}\right|+\left|\lambda_{n+1}-\lambda_{n}\right|\right),
$$

where $M_{1}$ is an appropriate constant such that

$$
M_{1} \geq \max \left\{\sup _{n \geq 1}\left\|B_{1} y_{n}\right\|, \sup _{n \geq 1}\left\|B_{2} x_{n}\right\|\right\} .
$$

Notice that

$$
\begin{aligned}
&\left\|x_{n+2}-x_{n+1}\right\| \\
& \leq \|\left(I-\alpha_{n+1} A\right)\left(W_{n+1} \rho_{n+1}-W_{n} \rho_{n}\right)-\left(\alpha_{n+1}-\alpha_{n}\right) A W_{n} \rho_{n} \\
&+\gamma\left(\alpha_{n+1}\left(f\left(x_{n+1}\right)-f\left(x_{n}\right)\right)+f\left(x_{n}\right)\left(\alpha_{n+1}-\alpha_{n}\right)\right) \| \\
& \leq\left(1-\alpha_{n+1} \bar{\gamma}\right)\left(\left\|\rho_{n+1}-\rho_{n}\right\|+\left\|W_{n+1} \rho_{n}-W_{n} \rho_{n}\right\|\right)+\left|\alpha_{n+1}-\alpha_{n}\right|\left\|A W_{n} \rho_{n}\right\| \\
&+\gamma\left(\alpha_{n+1} \alpha\left\|x_{n+1}-x_{n}\right\|+\left\|f\left(x_{n}\right)\right\|\left|\alpha_{n+1}-\alpha_{n}\right|\right) .
\end{aligned}
$$

Since $T_{i}$ and $U_{n, i}$ are nonexpansive, we have from (2.6) that

$$
\begin{aligned}
\left\|W_{n+1} \rho_{n}-W_{n} \rho_{n}\right\| & =\left\|\gamma_{1} T_{1} U_{n+1,2} \rho_{n}-\gamma_{1} T_{1} U_{n, 2} \rho_{n}\right\| \\
& \leq \gamma_{1}\left\|U_{n+1,2} \rho_{n}-U_{n, 2} \rho_{n}\right\| \\
& =\gamma_{1}\left\|\gamma_{2} T_{2} U_{u+1,3} \rho_{n}-\gamma_{2} T_{2} U_{n, 3} \rho_{n}\right\| \\
& \leq \gamma_{1} \gamma_{2}\left\|U_{u+1,3} \rho_{n}-U_{n, 3} \rho_{n}\right\| \\
& \leq \cdots \\
& \leq \gamma_{1} \gamma_{2} \cdots \gamma_{n}\left\|U_{n+1, n+1} \rho_{n}-U_{n, n+1} \rho_{n}\right\| \\
& \leq M_{2} \prod_{i=1}^{n} \gamma_{i},
\end{aligned}
$$

where $M_{2} \geq 0$ is an appropriate constant such that $\left\|U_{n+1, n+1} \rho_{n}-U_{n, n+1} \rho_{n}\right\| \leq M_{2}$ for each $n \geq 1$. Substituting (3.4) and (3.6) into (3.5), we arrive at

$$
\begin{aligned}
\left\|x_{n+2}-x_{n+1}\right\| \leq & \left(1-\alpha_{n+1}(\bar{\gamma}-\alpha \gamma)\right)\left\|x_{n+1}-x_{n}\right\| \\
& +M_{3}\left(\prod_{i=1}^{n} \gamma_{i}+\left|\alpha_{n+1}-\alpha_{n}\right|+\left|\lambda_{n+1}-\lambda_{n}\right|+\left|\eta_{n+1}-\eta_{n}\right|\right),
\end{aligned}
$$

where $M_{3}$ is an appropriate constant such that

$$
M_{3}=\max \left\{M_{1}, M_{2}, \gamma \sup _{n \geq 1}\left\{\left\|f\left(x_{n}\right)\right\|+\left\|A W_{n} \rho_{n}\right\|\right\}\right\} .
$$


From the restrictions (a) and (b), we obtain from Lemma 2.6 that

$$
\lim _{n \rightarrow \infty}\left\|x_{n+1}-x_{n}\right\|=0
$$

Notice that

$$
\begin{aligned}
\left\|x_{n+1}-W_{n} \rho_{n}\right\| & =\left\|P_{K}\left(\alpha_{n} \gamma f\left(x_{n}\right)+\left(I-\alpha_{n} A\right) W_{n} P_{K}\left(I-\lambda_{n} B_{1}\right) y_{n}\right)-P_{K}\left(W_{n} \rho_{n}\right)\right\| \\
& \leq \alpha_{n}\left\|\gamma f\left(x_{n}\right)-A W_{n} \rho_{n}\right\| .
\end{aligned}
$$

It follows from the restriction (a) that

$$
\lim _{n \rightarrow \infty}\left\|W_{n} \rho_{n}-x_{n+1}\right\|=0
$$

Notice that

$$
\begin{aligned}
\left\|y_{n}-p\right\|^{2} & \leq\left\|\left(x_{n}-p\right)-\eta_{n}\left(B_{2} x_{n}-B_{2} p\right)\right\|^{2} \\
& \leq\left\|x_{n}-p\right\|^{2}-2 \eta_{n} \mu_{2}\left\|B_{2} x_{n}-B_{2} p\right\|^{2}+\eta_{n}^{2}\left\|B_{2} x_{n}-B_{2} p\right\|^{2} \\
& =\left\|x_{n}-p\right\|^{2}+\left(\eta_{n}^{2}-2 \eta_{n} \mu_{2}\right)\left\|B_{2} x_{n}-B_{2} p\right\|^{2} .
\end{aligned}
$$

In a similar way, we find that

$$
\left\|\rho_{n}-p\right\|^{2} \leq\left\|x_{n}-p\right\|^{2}+\left(\lambda_{n}^{2}-2 \lambda \mu_{1}\right)\left\|B_{1} y_{n}-B_{1} p\right\|^{2} .
$$

On the other hand, we have

$$
\begin{aligned}
\left\|x_{n+1}-p\right\|^{2} & \leq\left\|\alpha_{n}\left(\gamma f\left(x_{n}\right)-A p\right)+\left(I-\alpha_{n} A\right)\left(W_{n} \rho_{n}-p\right)\right\|^{2} \\
& \leq\left(\alpha_{n}\left\|\gamma f\left(x_{n}\right)-A p\right\|+\left(1-\alpha_{n} \bar{\gamma}\right)\left\|\rho_{n}-p\right\|\right)^{2} \\
& \leq \alpha_{n}\left\|\gamma f\left(x_{n}\right)-A p\right\|^{2}+\left\|\rho_{n}-p\right\|^{2}+2 \alpha_{n}\left\|\gamma f\left(x_{n}\right)-A p\right\|\left\|\rho_{n}-p\right\| .
\end{aligned}
$$

Substituting (3.11) into (3.12) gives

$$
\begin{gathered}
\left\|x_{n+1}-p\right\|^{2} \leq \\
\alpha_{n}\left\|\gamma f\left(x_{n}\right)-A p\right\|^{2}+\left\|x_{n}-p\right\|^{2}+\left(\lambda_{n}^{2}-2 \lambda_{n} \mu_{1}\right)\left\|B_{1} y_{n}-B_{1} p\right\|^{2} \\
+2 \alpha_{n}\left\|\gamma f\left(x_{n}\right)-A p\right\|\left\|\rho_{n}-p\right\| .
\end{gathered}
$$

It follows from the restriction (c) that

$$
\begin{aligned}
& u\left(2 \mu_{1}-v\right)\left\|B_{1} y_{n}-B_{1} p\right\|^{2} \\
& \leq \alpha_{n}\left\|\gamma f\left(x_{n}\right)-A p\right\|^{2}+\left\|x_{n}-p\right\|^{2}-\left\|x_{n+1}-p\right\|^{2}+2 \alpha_{n}\left\|\gamma f\left(x_{n}\right)-A p\right\|\left\|\rho_{n}-p\right\| \\
& \leq \alpha_{n}\left\|\gamma f\left(x_{n}\right)-B p\right\|^{2}+\left(\left\|x_{n}-p\right\|+\left\|x_{n+1}-p\right\|\right)\left\|x_{n}-x_{n+1}\right\| \\
& \quad+2 \alpha_{n}\left\|\gamma f\left(x_{n}\right)-A p\right\|\left\|\rho_{n}-p\right\| .
\end{aligned}
$$

In view of the restriction (a), we obtain from (3.8) that

$$
\lim _{n \rightarrow \infty}\left\|B_{1} y_{n}-B_{1} p\right\|=0
$$


From (3.12), we also have

$$
\begin{aligned}
\left\|x_{n+1}-p\right\|^{2} \leq & \alpha_{n}\left\|\gamma f\left(x_{n}\right)-A p\right\|^{2}+\left\|y_{n}-p\right\|^{2} \\
& +2 \alpha_{n}\left\|\gamma f\left(x_{n}\right)-A p\right\|\left\|\rho_{n}-p\right\| .
\end{aligned}
$$

Combining (3.10) with (3.14), we arrive at

$$
\begin{aligned}
\left\|x_{n+1}-p\right\|^{2} \leq & \alpha_{n}\left\|\gamma f\left(x_{n}\right)-A p\right\|^{2}+\left\|x_{n}-p\right\|^{2}+\left(\eta_{n}^{2}-2 \eta_{n} \mu_{2}\right)\left\|B_{2} x_{n}-B_{2} p\right\|^{2} \\
& +2 \alpha_{n}\left\|\gamma f\left(x_{n}\right)-A p\right\|\left\|\rho_{n}-p\right\|,
\end{aligned}
$$

which implies from the restriction (c) that

$$
\begin{aligned}
& u\left(2 \mu_{2}-v\right)\left\|B_{2} x_{n}-B_{2} p\right\|^{2} \\
& \leq \alpha_{n}\left\|\gamma f\left(x_{n}\right)-A p\right\|^{2}+\left\|x_{n}-p\right\|^{2}-\left\|x_{n+1}-p\right\|^{2} \\
& \quad+2 \alpha_{n}\left\|\gamma f\left(x_{n}\right)-A p\right\|\left\|\rho_{n}-p\right\| \\
& \leq \alpha_{n}\left\|\gamma f\left(x_{n}\right)-A p\right\|^{2}+\left(\left\|x_{n}-p\right\|+\left\|x_{n+1}-p\right\|\right)\left\|x_{n}-x_{n+1}\right\| \\
& \quad+2 \alpha_{n}\left\|\gamma f\left(x_{n}\right)-A p\right\|\left\|\rho_{n}-p\right\| .
\end{aligned}
$$

In view of the restriction (a), we obtain from (3.8) that

$$
\lim _{n \rightarrow \infty}\left\|B_{2} x_{n}-B_{2} p\right\|=0
$$

On the other hand, we see from the firm expansivity of $P_{K}$ that

$$
\begin{aligned}
\left\|y_{n}-p\right\|^{2}= & \left\|P_{K}\left(I-\eta_{n} B_{2}\right) x_{n}-P_{K}\left(I-\eta_{n} B_{2}\right) p\right\|^{2} \\
\leq & \left\langle\left(I-\eta_{n} B_{2}\right) x_{n}-\left(I-\eta_{n} B_{2}\right) p, y_{n}-p\right\rangle \\
= & \frac{1}{2}\left(\left\|\left(I-\eta_{n} B_{2}\right) x_{n}-\left(I-\eta_{n} B_{2}\right) p\right\|^{2}+\left\|y_{n}-p\right\|^{2}\right. \\
& \left.-\left\|\left(I-\eta_{n} B_{2}\right) x_{n}-\left(I-\eta_{n} B_{2}\right) p-\left(y_{n}-p\right)\right\|^{2}\right) \\
\leq & \frac{1}{2}\left(\left\|x_{n}-p\right\|^{2}+\left\|y_{n}-p\right\|^{2}-\left\|\left(x_{n}-y_{n}\right)-\eta_{n}\left(B_{2} x_{n}-B_{2} p\right)\right\|^{2}\right) \\
= & \frac{1}{2}\left(\left\|x_{n}-p\right\|^{2}+\left\|y_{n}-p\right\|^{2}-\left\|x_{n}-y_{n}\right\|^{2}-\eta_{n}^{2}\left\|B_{2} x_{n}-B_{2} p\right\|^{2}\right. \\
& \left.+2 \eta_{n}\left\langle x_{n}-y_{n}, B_{2} x_{n}-B_{2} p\right\rangle\right),
\end{aligned}
$$

which yields

$$
\left\|y_{n}-p\right\|^{2} \leq\left\|x_{n}-p\right\|^{2}-\left\|x_{n}-y_{n}\right\|^{2}+2 \eta_{n}\left\|x_{n}-y_{n}\right\|\left\|B_{2} x_{n}-B_{2} p\right\| .
$$

In the same way, we can obtain that

$$
\left\|\rho_{n}-p\right\|^{2} \leq\left\|x_{n}-p\right\|^{2}-\left\|\rho_{n}-y_{n}\right\|^{2}+2 \lambda_{n}\left\|\rho_{n}-y_{n}\right\|\left\|B_{1} y_{n}-B_{1} p\right\| .
$$


Substituting (3.16) into (3.14) yields

$$
\begin{aligned}
\left\|x_{n+1}-p\right\|^{2} \leq & \alpha_{n}\left\|\gamma f\left(x_{n}\right)-A p\right\|^{2}+\left\|x_{n}-p\right\|^{2}-\left\|x_{n}-y_{n}\right\|^{2} \\
& +2 \eta_{n}\left\|x_{n}-y_{n}\right\|\left\|B_{2} x_{n}-B_{2} p\right\|+2 \alpha_{n}\left\|\gamma f\left(x_{n}\right)-A p\right\|\left\|\rho_{n}-p\right\| .
\end{aligned}
$$

It follows that

$$
\begin{aligned}
\left\|x_{n}-y_{n}\right\|^{2} \leq & \alpha_{n}\left\|\gamma f\left(x_{n}\right)-A p\right\|^{2}+\left\|x_{n}-p\right\|^{2}-\left\|x_{n+1}-p\right\|^{2} \\
& +2 \eta_{n}\left\|x_{n}-y_{n}\right\|\left\|B_{2} x_{n}-B_{2} p\right\|+2 \alpha_{n}\left\|\gamma f\left(x_{n}\right)-A p\right\|\left\|\rho_{n}-p\right\| \\
\leq & \alpha_{n}\left\|\gamma f\left(x_{n}\right)-A p\right\|^{2}+\left(\left\|x_{n}-p\right\|+\left\|x_{n+1}-p\right\|\right)\left\|x_{n+1}-x_{n}\right\| \\
& +2 \eta_{n}\left\|x_{n}-y_{n}\right\|\left\|B_{2} x_{n}-B_{2} p\right\|+2 \alpha_{n}\left\|\gamma f\left(x_{n}\right)-A p\right\|\left\|\rho_{n}-p\right\| .
\end{aligned}
$$

In view of (3.7) and (3.15), we see from the restriction (a) that

$$
\lim _{n \rightarrow \infty}\left\|x_{n}-y_{n}\right\|=0
$$

Similarly, we can obtain that

$$
\lim _{n \rightarrow \infty}\left\|\rho_{n}-y_{n}\right\|=0
$$

Observe that

$$
\left\|\rho_{n}-W_{n} \rho_{n}\right\| \leq\left\|y_{n}-\rho_{n}\right\|+\left\|x_{n}-y_{n}\right\|+\left\|x_{n}-x_{n+1}\right\|+\left\|x_{n+1}-W_{n} \rho_{n}\right\| .
$$

It follows from (3.7), (3.9), (3.18) and (3.19) that

$$
\lim _{n \rightarrow \infty}\left\|W_{n} \rho_{n}-\rho_{n}\right\|=0
$$

From Lemma 2.7, we have $\left\|W \rho_{n}-W_{n} \rho_{n}\right\| \rightarrow 0$ as $n \rightarrow \infty$. This in turn implies that

$$
\lim _{n \rightarrow \infty}\left\|W \rho_{n}-\rho_{n}\right\|=0
$$

Next, we show that

$$
\limsup _{n \rightarrow \infty}\left\langle\gamma f\left(x^{*}\right)-A x^{*}, x_{n}-x^{*}\right\rangle \leq 0 .
$$

To show it, we choose a subsequence $\left\{x_{n_{i}}\right\}$ of $\left\{x_{n}\right\}$ such that

$$
\limsup _{n \rightarrow \infty}\left\langle\gamma f\left(x^{*}\right)-A x^{*}, x_{n}-x^{*}\right\rangle=\lim _{i \rightarrow \infty}\left\langle\gamma f\left(x^{*}\right)-A x^{*}, x_{n_{i}}-x^{*}\right\rangle .
$$

As $\left\{x_{n_{i}}\right\}$ is bounded, we have that a subsequence $\left\{x_{n_{i_{j}}}\right\}$ of $\left\{x_{n_{i}}\right\}$ converges weakly to $p$. We may assume, without loss of generality, that $x_{n_{i}} \rightarrow p$. From (3.18) and (3.19), we also 
have $y_{n_{i}} \rightarrow p$ and $z_{n_{i}} \rightarrow p$, respectively. Notice that $p \in F$. Indeed, let us first show that $p \in V I\left(K, B_{1}\right)$. Put

$$
S w= \begin{cases}B_{1} v+N_{K} v, & v \in K, \\ \emptyset, & v \notin K .\end{cases}
$$

Then $S$ is maximal monotone. Let $(v, w) \in G(S)$. Since $w-B_{1} v \in N_{K} v$ and $\rho_{n} \in K$, we have

$$
\left\langle v-\rho_{n}, w-B_{1} v\right\rangle \geq 0
$$

On the other hand, we have from $\rho_{n}=P_{K}\left(I-\lambda_{n} B_{1}\right) y_{n}$ that

$$
\left\langle v-\rho_{n}, \rho_{n}-\left(I-\lambda_{n} B_{1}\right) y_{n}\right\rangle \geq 0
$$

and hence

$$
\left\langle v-\rho_{n}, \frac{\rho_{n}-y_{n}}{\lambda_{n}}+B_{1} y_{n}\right\rangle \geq 0 .
$$

It follows that

$$
\begin{aligned}
\left\langle v-\rho_{n_{i}}, w\right\rangle & \geq\left\langle v-\rho_{n_{i}}, B_{1} v\right\rangle \\
& \geq\left\langle v-\rho_{n_{i}}, B_{1} v\right\rangle-\left\langle v-\rho_{n_{i}}, \frac{\rho_{n_{i}}-y_{n_{i}}}{\lambda_{n_{i}}}+B_{1} y_{n_{i}}\right\rangle \\
& \geq\left\langle v-\rho_{n_{i}}, B_{1} v-\frac{\rho_{n_{i}}-y_{n_{i}}}{\lambda_{n_{i}}}-B_{1} y_{n_{i}}\right\rangle \\
& =\left\langle v-\rho_{n_{i}}, B_{1} v-B_{1} \rho_{n_{i}}\right\rangle+\left\langle v-\rho_{n_{i}}, B_{1} \rho_{n_{i}}-B_{1} y_{n_{i}}\right\rangle-\left\langle v-\rho_{n_{i}}, \frac{\rho_{n_{i}}-y_{n_{i}}}{\lambda_{n_{i}}}\right\rangle \\
& \geq\left\langle v-\rho_{n_{i}}, B_{1} \rho_{n_{i}}-B_{1} y_{n_{i}}\right\rangle-\left\langle v-\rho_{n_{i}}, \frac{\rho_{n_{i}}-y_{n_{i}}}{\lambda_{n_{i}}}\right\rangle,
\end{aligned}
$$

which implies that $\langle v-p, w\rangle \geq 0$. We have $p \in B_{1}^{-1} 0$ and hence $p \in V I\left(K, B_{1}\right)$. In a similar way, we can show $p \in V I\left(K, B_{2}\right)$. Next, let us show $p \in \bigcap_{i=1}^{\infty} F\left(T_{i}\right)$. Since Hilbert spaces satisfy Opial's condition, we see from (3.21) that

$$
\begin{aligned}
\liminf _{i \rightarrow \infty}\left\|\rho_{n_{i}}-p\right\| & <\liminf _{i \rightarrow \infty}\left\|\rho_{n_{i}}-W p\right\| \\
& =\liminf _{i \rightarrow \infty}\left\|\rho_{n_{i}}-W \rho_{n_{i}}+W \rho_{n_{i}}-W p\right\| \\
& \leq \liminf _{i \rightarrow \infty}\left\|W \rho_{n_{i}}-W p\right\| \\
& \leq \liminf _{i \rightarrow \infty}\left\|\rho_{n_{i}}-p\right\|,
\end{aligned}
$$

which derives a contradiction. Thus, we have $p \in F(W)=\bigcap_{i=1}^{\infty} F\left(T_{i}\right)$. From Lemma 2.5, we see that there exists a unique $x^{*}$ such that $x^{*}=P_{F}(\gamma f+(I-A)) x^{*}$. It follows that

$$
\limsup _{n \rightarrow \infty}\left\langle\gamma f\left(x^{*}\right)-A x^{*}, x_{n}-x^{*}\right\rangle=\left\langle\gamma f\left(x^{*}\right)-A x^{*}, p-x^{*}\right\rangle \leq 0
$$


That is, (3.22) holds. It follows from Lemma 2.3 that

$$
\begin{aligned}
\left\|x_{n+1}-x^{*}\right\|^{2} \leq & \left\|\alpha_{n}\left(\gamma f\left(x_{n}\right)-A x^{*}\right)+\left(I-\alpha_{n} A\right)\left(W_{n} \rho_{n}-x^{*}\right)\right\|^{2} \\
\leq & \left(1-\alpha_{n} \bar{\gamma}\right)^{2}\left\|W_{n} \rho_{n}-x^{*}\right\|^{2}+2 \alpha_{n}\left\langle\gamma f\left(x_{n}\right)-A x^{*}, x_{n+1}-x^{*}\right\rangle \\
\leq & \left(1-\alpha_{n} \bar{\gamma}\right)^{2}\left\|x_{n}-x^{*}\right\|^{2}+\alpha \gamma \alpha_{n}\left(\left\|x_{n}-x^{*}\right\|^{2}+\left\|x_{n+1}-x^{*}\right\|^{2}\right) \\
& +2 \alpha_{n}\left\langle\gamma f\left(x^{*}\right)-A x^{*}, x_{n+1}-x^{*}\right\rangle .
\end{aligned}
$$

It follows that

$$
\begin{aligned}
\left\|x_{n+1}-x^{*}\right\|^{2} \leq & \frac{\left(1-\alpha_{n} \bar{\gamma}\right)^{2}+\alpha_{n} \gamma \alpha}{1-\alpha_{n} \gamma \alpha}\left\|x_{n}-x^{*}\right\|^{2}+\frac{2 \alpha_{n}}{1-\alpha_{n} \gamma \alpha}\left\langle\gamma f\left(x^{*}\right)-A x^{*}, x_{n+1}-x^{*}\right\rangle \\
= & \frac{\left(1-2 \alpha_{n} \bar{\gamma}+\alpha_{n} \alpha \gamma\right)}{1-\alpha_{n} \gamma \alpha}\left\|x_{n}-x^{*}\right\|^{2}+\frac{\alpha_{n}^{2} \bar{\gamma}^{2}}{1-\alpha_{n} \gamma \alpha}\left\|x_{n}-x^{*}\right\|^{2} \\
& +\frac{2 \alpha_{n}}{1-\alpha_{n} \gamma \alpha}\left\langle\gamma f\left(x^{*}\right)-A x^{*}, x_{n+1}-x^{*}\right\rangle \\
\leq & {\left[1-\frac{2 \alpha_{n}(\bar{\gamma}-\alpha \gamma)}{1-\alpha_{n} \gamma \alpha}\right]\left\|x_{n}-x^{*}\right\|^{2} } \\
& +\frac{2 \alpha_{n}(\bar{\gamma}-\alpha \gamma)}{1-\alpha_{n} \gamma \alpha}\left(\frac{1}{\bar{\gamma}-\alpha \gamma}\left\langle\gamma f\left(x^{*}\right)-A x^{*}, x_{n+1}-x^{*}\right\rangle+\frac{\alpha_{n} \bar{\gamma}^{2}}{2(\bar{\gamma}-\alpha \gamma)} M_{4}\right)
\end{aligned}
$$

where $M_{4}$ is an appropriate constant such that $M_{4} \geq \sup _{n \geq 1}\left\|x_{n}-x^{*}\right\|^{2}$. Put $b_{n}=\frac{2 \alpha_{n}(\bar{\gamma}-\alpha \gamma)}{1-\alpha_{n} \alpha \gamma}$ and $c_{n}=\frac{1}{\bar{\gamma}-\alpha \gamma}\left\langle\gamma f\left(x^{*}\right)-A x^{*}, x_{n+1}-x^{*}\right\rangle+\frac{\alpha_{n} \bar{\gamma}^{2}}{2(\bar{\gamma}-\alpha \gamma)} M_{4}$. That is,

$$
\left\|x_{n+1}-x^{*}\right\|^{2} \leq\left(1-b_{n}\right)\left\|x_{n}-x^{*}\right\|+b_{n} c_{n}
$$

In view of the restrictions (a) and (b), we see from (3.22) that

$$
\lim _{n \rightarrow \infty} b_{n}=0, \quad \sum_{n=1}^{\infty} b_{n}=\infty \quad \text { and } \quad \limsup _{n \rightarrow \infty} c_{n} \leq 0 .
$$

Apply Lemma 2.6 to (3.23) to conclude that $x_{n} \rightarrow x^{*}$ as $n \rightarrow \infty$. This completes the proof.

If $B_{2}=0$, the zero mapping, then Theorem 3.1 is reduced to the following.

Corollary 3.2 Let $K$ be a nonempty, closed, and convex subset of a real Hilbert space $H$. Let $B_{1}: K \rightarrow H$ be $\mu_{1}$-inverse-strongly monotone mappings and $f: K \rightarrow K$ be an $\alpha$-contraction. Let $A: K \rightarrow K$ be a strongly positive linear bounded self-adjoint operator with the coeffcient $\bar{\gamma}>0$. Assume that $0<\gamma<\bar{\gamma} / \alpha$. Let $\left\{x_{n}\right\}$ be a sequence generated in the following iterative algorithm:

$$
\left\{\begin{array}{l}
x_{1} \in K, \\
x_{n+1}=P_{K}\left(\alpha_{n} \gamma f\left(x_{n}\right)+\left(I-\alpha_{n} A\right) W_{n} P_{K}\left(I-\lambda_{n} B_{1}\right) x_{n}\right), \quad n \geq 1,
\end{array}\right.
$$


where $P_{K}$ is the metric projection from $H$ onto $K, W_{n}$ is a mapping defined by (2.5), $\left\{\alpha_{n}\right\}$ is a real number sequence in $(0,1)$, and $\left\{\lambda_{n}\right\}$ is a positive real number sequence. Assume that $F=\bigcap_{i=1}^{\infty} F\left(T_{i}\right) \cap V I\left(K, B_{1}\right) \neq \emptyset$ and the following restrictions are satisfied:

(a) $\lim _{n \rightarrow \infty} \alpha_{n}=0, \sum_{n=1}^{\infty} \alpha_{n}=\infty$, and $\sum_{n=1}^{\infty}\left|\alpha_{n+1}-\alpha_{n}\right| \leq \infty$;

(b) $\sum_{n=1}^{\infty}\left|\lambda_{n+1}-\lambda_{n}\right|<\infty$;

(c) $\left\{\lambda_{n}\right\} \subset[u, v]$, where $0<u<v<2 \mu_{1}$.

Then the sequence $\left\{x_{n}\right\}$ strongly converges to $x^{*} \in F$, where $x^{*}=P_{F}(\gamma f+(I-A)) x^{*}$.

Remark 3.3 Corollary 3.2 includes the corresponding results in Iiduka and Takahashi [36] as a special case.

As an application of our main results, we consider another class of important nonlinear operators: strict pseudocontractions.

Recall that a mapping $S: K \rightarrow K$ is said to be a $\kappa$-strict pseudocontraction if there exists a constant $\kappa \in[0,1)$ such that

$$
\|S x-S y\|^{2} \leq\|x-y\|^{2}+\kappa\|(I-S) x-(I-S) y\|^{2}, \quad \forall x, y \in K .
$$

It is easy to see that the class of $\kappa$-strict pseudocontractions strictly includes the class of nonexpansive mappings as a special case.

Putting $B=I-S$, where $S: K \rightarrow K$ is a $\kappa$-strict pseudocontraction, we know that $B$ is $\frac{1-\kappa}{2}$-inverse-strongly monotone; see [43] and the references therein.

Corollary 3.4 Let $H$ be a real Hilbert space and $K$ be a nonempty closed convex subset of $H$. Let $S_{i}: K \rightarrow K$ be $\kappa_{i}$-inverse-strongly monotone mappings for each $i=1,2$ and $f: K \rightarrow K$ be an $\alpha$-contraction. Let $A: K \rightarrow K$ be a strongly positive linear bounded self-adjoint operator with the coefficient $\bar{\gamma}>0$. Assume that $0<\gamma<\bar{\gamma} / \alpha$. Let $\left\{x_{n}\right\}$ be a sequence generated in the following iterative process:

$$
\left\{\begin{array}{l}
x_{1} \in K, \\
y_{n}=\left(1-\eta_{n}\right) x_{n}+\eta_{n} S_{2} x_{n}, \\
x_{n+1}=P_{K}\left(\alpha_{n} \gamma f\left(x_{n}\right)+\left(I-\alpha_{n} A\right) W_{n}\left(\left(1-\lambda_{n}\right) y_{n}+\lambda_{n} S_{1} y_{n}\right)\right), \quad n \geq 1,
\end{array}\right.
$$

where $P_{K}$ is the metric projection from $H$ onto $K, W_{n}$ is a mapping defined by (2.5), $\left\{\alpha_{n}\right\}$ is a real number sequence in $(0,1)$, and $\left\{\lambda_{n}\right\},\left\{\eta_{n}\right\}$ are two positive real number sequences. Assume that $F=\bigcap_{i=1}^{\infty} F\left(T_{i}\right) \cap F\left(S_{1}\right) \cap F\left(S_{2}\right) \neq \emptyset$ and the following restrictions are satisfied:

(a) $\lim _{n \rightarrow \infty} \alpha_{n}=0, \sum_{n=1}^{\infty} \alpha_{n}=\infty$, and $\sum_{n=1}^{\infty}\left|\alpha_{n+1}-\alpha_{n}\right| \leq \infty$;

(b) $\sum_{n=1}^{\infty}\left|\eta_{n+1}-\eta_{n}\right|<\infty, \sum_{n=1}^{\infty}\left|\lambda_{n+1}-\lambda_{n}\right|<\infty$;

(c) $\left\{\eta_{n}\right\},\left\{\lambda_{n}\right\} \subset[u, v]$, where $0<u<v<2 \min \left\{\mu_{1}, \mu_{2}\right\}$.

Then the sequence $\left\{x_{n}\right\}$ strongly converges to $x^{*} \in F$, where $x^{*}=P_{F}(\gamma f+(I-A)) x^{*}$.

Proof Put $B_{1}=I-S_{1}$ and $B_{2}=I-S_{2}$. Then $B_{1}$ is $\left(1-\kappa_{1}\right) / 2$-inverse-strongly monotone and $B_{2}$ is $\left(1-\kappa_{2}\right) / 2$-inverse-strongly monotone, respectively. We have $F\left(S_{1}\right)=V I\left(K, B_{1}\right), F\left(S_{2}\right)=$ $V I\left(K, B_{2}\right), P_{K}\left(I-\lambda_{n} B_{1}\right) y_{n}=\left(1-\lambda_{n}\right) y_{n}+\lambda_{n} T_{1} y_{n}$ and $P_{K}\left(I-\eta_{n} B_{2}\right) x_{n}=\left(1-\eta_{n}\right) x_{n}+\eta_{n} T_{2} x_{n}$. The desired conclusion can be immediately obtained from Theorem 3.1 . 


\section{Competing interests}

The authors declare that they have no competing interests.

\section{Authors' contributions}

Both authors participated in the design of this work and performed equally. Both authors read and approved the final manuscript.

\section{Author details}

${ }^{1}$ Department of Mathematics, Hangzhou Normal University, Hangzhou, 310036, China. ${ }^{2}$ Department of Mathematics, School of Science, Beijing Jiaotong University, Beijing, 100044, China. ${ }^{3}$ Department of Mathematics, Shijiazhuang University, Shijiazhuang, 050035, China.

\section{Acknowledgements}

This research was supported by the Natural Science Foundation of Hebei Province (A2010001943), the Science Foundation of Shijiazhuang Science and Technology Bureau (121130971) and the Science Foundation of Beijing Jiaotong University (2011YJS075).

Received: 31 December 2012 Accepted: 21 February 2013 Published: 25 March 2013

\section{References}

1. Bauschke, HH, Borwein, JM: On projection algorithms for solving convex feasibility problems. SIAM Rev. 38, 367-426 (1996)

2. Qin, X, Cho, SY, Kang, SM: Common fixed points of a pair of non-expansive mappings with applications to convex feasibility problems. Glasg. Math. J. 52, 241-252 (2010)

3. Kotzer, T, Cohen, N, Shamir, J: Image restoration by a novel method of parallel projection onto constraint sets. Optim. Lett. 20, 1772-1774 (1995)

4. Hao, $Y$, Wang, $X$, Tong, A: Weak and strong convergence theorems for two finite families of asymptotically nonexpansive mappings in Banach spaces. Adv. Fixed Point Theory 2, 417-432 (2012)

5. Cho, SY, Kang, SM: Some results on asymptotically hemi-pseudocontractive mappings in the intermediate sense. Fixed Point Theory Appl. 13, 153-164 (2012)

6. Qin, X, Cho, SY, Kang, SM: On hybrid projection methods for asymptotically quasi- $\phi$-nonexpansive mappings. Appl. Math. Comput. 215, 3874-3883 (2010)

7. Manro, S, Kumar, S, Bhatia, SS: Common fixed point theorems in intuitionistic fuzzy metric spaces using occasionally weakly compatible maps. J. Math. Comput. Sci. 2, 73-81 (2012)

8. Lv, S, Wu, C: Convergence of iterative algorithms for a generalized variational inequality and a nonexpansive mapping. Eng. Math. Lett. 1, 44-57 (2012)

9. Qin, X, Su, Y: Strong convergence theorems for relatively nonexpansive mappings in a Banach space. Nonlinear Anal. 67, 1958-1965 (2007)

10. Cho, SY, Kang, SM: Hybrid projection algorithms for treating common fixed points of a family of demicontinuous pseudocontractions. Appl. Math. Lett. 25, 854-857 (2012)

11. Qin, $X$, Cho, YJ, Kang, SM, Zhou, H: Convergence of a modified Halpern-type iteration algorithm for quasi- $\boldsymbol{\phi}$-nonexpansive mappings. Appl. Math. Lett. 22, 1051-1055 (2009)

12. Genel, A, Lindenstruss, J: An example concerning fixed points. Isr. J. Math. 22, 81-86 (1975)

13. Khan, MA, Yannelis, NC: Equilibrium Theory in Infinite Dimensional Spaces. Springer, New York (1991)

14. Combettes, PL: The Convex Feasibility Problem in Image Recovery. In: Hawkes, P (ed.) Advances in Imaging and Electron Physics, vol. 95, pp. 155-270. Academic Press, New York (1996)

15. Dautray, R, Lions, JL: Mathematical Analysis and Numerical Methods for Science and Technology, vol. 1. Springer, New York (1988)

16. Dautray, R, Lions, JL: Mathematical Analysis and Numerical Methods for Science and Technology, vol. 2. Springer, New York (1989)

17. Dautray, R, Lions, JL: Mathematical Analysis and Numerical Methods for Science and Technology, vol. 3. Springer, New York (1990)

18. Dautray, R, Lions, JL: Mathematical Analysis and Numerical Methods for Science and Technology, vol. 4. Springer, New York (1991)

19. Dautray, R, Lions, JL: Mathematical Analysis and Numerical Methods for Science and Technology, vol. 5. Springer, New York (1992)

20. Dautray, R, Lions, JL: Mathematical Analysis and Numerical Methods for Science and Technology, vol. 6. Springer, New York (1993)

21. Fattorini, HO: Infinite-Dimensional Optimization and Control Theory. Cambridge University Press, Cambridge (1999)

22. Reich, S: Strong convergence theorems for resolvents of accretive operators in Banach spaces. J. Math. Anal. Appl. 75, 287-292 (1980)

23. Qin, X, Su, Y: Approximation of a zero point of accretive operator in Banach spaces. J. Math. Anal. Appl. 329, 415-424 (2007)

24. Rockafellar, RT: On the maximality of sums of nonlinear monotone operators. Trans. Am. Math. Soc. 149, 75-88 (1970)

25. Xu, HK: An iterative approach to quadratic optimization. J. Optim. Theory Appl. 116, 659-678 (2003)

26. Marino, G, Xu, HK: A general iterative method for nonexpansive mappings in Hilbert spaces. J. Math. Anal. Appl. 318 43-52 (2006)

27. Qin, X, Shang, M, Su, Y: Strong convergence of a general iterative algorithm for equilibrium problems and variational inequality problems. Math. Comput. Model. 48, 1033-1046 (2008)

28. Cho, SY, Kang, SM: Approximation of fixed points of pseudocontraction semigroups based on a viscosity iterative process. Appl. Math. Lett. 24, 224-228 (2011)

29. Zegeye, $\mathrm{H}$, Shahzad, N: Strong convergence theorem for a common point of solution of variational inequality and fixed point problem. Adv. Fixed Point Theory 4, 374-397 (2012) 
30. Luo, H, Wang, Y: Iterative approximation for the common solutions of a infinite variational inequality system for inverse-strongly accretive mappings. J. Math. Comput. Sci. 2, 1660-1670 (2012)

31. Cho, SY, Kang, SM: Approximation of common solutions of variational inequalities via strict pseudocontractions. Acta Math. Sci. 32, 1607-1618 (2012)

32. Ye, J, Huang, J: Strong convergence theorems for fixed point problems and generalized equilibrium problems of three relatively quasi-nonexpansive mappings in Banach spaces. J. Math. Comput. Sci. 1, 1-18 (2011)

33. Qin, X, Cho, SY, Kang, SM: Strong convergence of shrinking projection methods for quasi- $\phi$-nonexpansive mappings and equilibrium problems. J. Comput. Appl. Math. 234, 750-760 (2010)

34. Yang, S, Li, W: Iterative solutions of a system of equilibrium problems in Hilbert spaces. Adv. Fixed Point Theory 1 , 15-26 (2011)

35. Takahashi, W, Toyoda, M: Weak convergence theorems for nonexpansive mappings and monotone mappings. J. Optim. Theory Appl. 118, 417-428 (2003)

36. liduka, $\mathrm{H}$, Takahashi, W: Strong convergence theorems for nonexpansive mappings and inverse-strongly monotone mappings. Nonlinear Anal. 61, 341-350 (2005)

37. Hao, Y: On variational inclusion and common fixed point problems in Hilbert spaces with applications. Appl. Math. Comput. 217, 3000-3010 (2010)

38. Qin, X, Cho, SY, Kang, SM: Convergence theorems of common elements for equilibrium problems and fixed point problems in Banach spaces. J. Comput. Appl. Math. 225, 20-30 (2009)

39. Chang, SS, Lee, HWJ, Chan, CK: A new method for solving equilibrium problem fixed point problem and variational inequality problem with application to optimization. Nonlinear Anal. 70, 3307-3319 (2009)

40. Qin, X, Cho, YJ, Kang, SM: Viscosity approximation methods for generalized equilibrium problems and fixed point problems with applications. Nonlinear Anal. 72, 99-112 (2010)

41. Shimoji, K, Takahashi, W: Strong convergence to common fixed points of infinite nonexpansive mappings and applications. Taiwan. J. Math. 5, 387-404 (2001)

42. Liu, L: Ishikawa-type and Mann-type iterative processes with errors for constructing solutions of nonlinear equations involving m-accretive operators in Banach spaces. Nonlinear Anal. 34, 307-317 (1998)

43. Browder, FE, Petryshyn, WV: Construction of fixed points of nonlinear mappings in Hilbert space. J. Math. Anal. Appl. 20, 197-228 (1967)

doi:10.1186/1687-1812-2013-67

Cite this article as: Qing and Shang: Convergence of an extragradient-like iterative algorithm for monotone mappings and nonexpansive mappings. Fixed Point Theory and Applications 2013 2013:67.

\section{Submit your manuscript to a SpringerOpen ${ }^{\ominus}$ journal and benefit from:}

- Convenient online submission

- Rigorous peer review

- Immediate publication on acceptance

- Open access: articles freely available online

- High visibility within the field

- Retaining the copyright to your article 